\title{
Brain Stimulation, Alternative Medicine, and Impulsivity and Compulsivity
}

By Eric Hollander, MD

I am pleased to announce that CNS Spectrums is launching two new columns this month.

Stefano Pallanti, MD, PhD, introduces the timely new column "Brain Stimulation," which synthesizes the practical applications of the latest brain stimulation techniques and relates these findings to new understandings in neuropsychiatry. This column will be co-written by Thomas E. Schlaepfer, MD.

Uriel Halbreich, MD, initiates his new column, "The Well-Rounded Brain," which systematically and critically reviews the evidence and myths of complementary and alternative medicine. This too is especially timely, since our patients are already taking such treatments, and clinicians need to understand the knowledge and evidence base for such approaches.

Tics and Tourette syndrome (TS) are common comorbidities of patients diagnosed with ADHD. Stimulant medications for ADHD have been associated with tic exacerbations, thus limiting their utility in this patient subgroup. Atomoxetine has been explored as an alternative treatment as one of the few non-stimulants available to treat ADHD. There are, however, few case studies describing the impact of atomoxetine on tics. Jessica Sears, MD, and Nitin C. Patel, MD, present a unique case of a patient, without any prior history of a movement disorder, who developed tics following a single dose of atomoxetine, that did not improve until interventional therapy was initiated. A goal of future treatments may be to enhance prefrontal dopamine without increasing nigrostriatal dopamine in patients with tics and ADHD.

Kelsie T. Forbush, MA, and colleagues examine neuropsychological and personality characteristics in pathological gambling disorder (PG), a condition which has been associated with fronto-temporal dysfunction and maladaptive personality traits, such as impulsivity and novelty seeking. Subjects with PG had impaired decision making on the lowa Gambling Task, elevated levels of impulsivity, novelty seeking, and harm avoidance and lower levels of selfdirectedness and cooperativeness. Of interest, personality traits were better predictors than neuropsychological characteristics of whether someone has PG. However, it is of interest that there may be multiple developmental trajectories to impulsivity, including long-term trait impulsivity as well as neurocognitive deficits associated with impaired decision making and frontal disinhibition.

In clinical samples, body dysmorphic disorder (BDD) is associated with substantial suffering and reduced quality of life. Limited surveys report widely varying prevalence estimates. To better establish the prevalence of BDD, Lorrin $M$. Koran, MD, and colleagues conducted a nationwide prevalence survey in the United States. They found that the estimated prevalence of Diagnostic and Statistical Manual of Mental Disorders, Fourth Edition BDD among respondents was $2.4 \%$ (49 out of 2,048) (by gender: $2.5 \%$ for women, $2.2 \%$ for men), exceeding the

Dr. Hollander is the editor of this journal, Esther and Joseph Klingenstein Professor and Chairman of Psychiatry at the Mount Sinai School of Medicine, and director of the Seaver and New York Autism Center of Excellence in New York Ciry. 
prevalence of schizophrenia and bipolar I disorder and about that of generalized anxiety disorder. BDD prevalence decreased after 44 years of age and a larger proportion of BDD respondents were never married. Of those meeting $D S M$ $I V$ criteria for BDD, $90 \%$ (45 out of 49 ) met the $D S M-I V$ distress criterion, and $51 \%$ (25 out of 49) met the interference-with-functioning criterion. A study using clinically valid interviews is needed to evaluate these results. These studies could inform treatment by documenting rates of seeking treatment from various sources, suicide attempt rates, and the prevalence of comorbid conditions. However, these type of studies suggest that obsessive-compulsive spectrum disorders, such as BDD, may be common and disabling, are clearly understudied, and should be the focus of increased research funding.

Yukiko Kano, MD, and colleagues conducted a study to explore possible causes of rage attacks as well as clinically significant aggressive symptoms in Japanese adolescents with TS. The subjects included 29 adolescents ( 23 males, 6 females; mean age: 13.5 years). Approximately $62 \%$ were diagnosed with TS alone and $37.9 \%$ with TS and comorbidities, including attention-deficit/hyperactivity disorder (ADHD) and obsessive-compulsive disorder (OCD). Over $44 \%$ had clinically significant aggressive symptoms. No differences were found in age, gender, psychiatric comorbidities, or concurrent medication in the aggressive and nonaggressive subjects. There were more anxious/ depressed, thought problem, aggressive, internalizing, and externalizing symptom severity in the aggressive group. Both rage attacks and clinically significant aggressive symptoms were common problems in Japanese TS youth. Psychiatric morbidity seems associated with impulsive-aggressive symptoms. Subjects with involvement of multiple symptom domains, including tics, ADHD, and $O C D$, have a more complex and heterogeneous presentation, which has important implications for course of illness and treatment response.

This issue addresses the study of methods such as brain stimulation and alternative medicine as well as the study of conditions such as the common, complex, and heterogenous presentation of impulsive and compulsive disorders that are much in the news these days and in need of further exploration. CNS

\section{CNS SPECTRUMS \\ Depression, Antidepressants, and Bone Loss-An Interview with Susan Diem, MD, MPH, is currently available as an audio PsychCast ${ }^{\text {MM }}$ at wWW.cnsspectrums.com.}

If you have any questions and/or comments regarding the interview with Susan Diem, MD, MPH, please submit a "Letter to the Editor" to Eric Hollander, MD, at vi@mblcommunications.com.

Additional PsychCasts ${ }^{\top M}$ include, "An Update on the Sexual Side Effects of Medications" with Anita H. Clayton, MD, "Neuromodulation" with Philip G. Janicak, MD, "Examining the Strengths and Limitations of Generic Formulations of Antidepressants" with Michael E. Thase, MD, and "Bipolar Disorder" with Joseph F. Goldberg, MD, among many others. Please visit www.cnsspectrums.com. 